\title{
Prevalence of Hepatitis B Reactivation Among Chinese Individuals With Chronic Hepatitis C Treated With Pan-Oral Direct-Acting Antivirals
}

\author{
Shou-Wu Lee ${ }^{a, b, ~ d}$, Teng-Yu Lee ${ }^{a, b}$, Sheng-Shun Yang ${ }^{a, ~ c}$, Yen-Chun Peng ${ }^{a, c}$, \\ Hong-Zen Yeh ${ }^{\mathrm{a}, \mathrm{c}}$, Chi-Sen Chang, ${ }^{\mathrm{a}} \mathrm{b}$
}

\begin{abstract}
Background: Clearance of hepatitis $\mathrm{C}$ virus (HCV) has been reported to induce the reactivation of hepatitis B virus (HBV). The aim of this study was to investigate the rate of $\mathrm{HBV}$ reactivation in $\mathrm{HCV}$-infected Chinese patients who received treatment with pan-oral direct-acting antivirals (DAAs).
\end{abstract}

Methods: Data from HCV subjects receiving oral DAA therapy were retrospectively collected from October 2015 to May 2017. Patients who were seropositive for $\mathrm{HBsAg}$ or anti-HBc were enrolled. The efficacy of DAAs, including end-of-treatment virologic response (ETVR) and sustained virologic response (SVR) 12, was recorded. HBV virologic reactivation was defined as a reappearance of HBsAg, or increased HBV DNA by at least one $\log _{10} \mathrm{IU} / \mathrm{mL}$. $\mathrm{HBV}$ clinical reactivation was defined as virologic reactivation and serum alanine aminotransferase (ALT) over two-fold of the upper limit of normal.

Results: There were $11(7.2 \%)$ cases and $53(34.6 \%)$ cases in the HBsAg group and the anti-HBc group among all 153 subjects. All individuals achieved ETVR and SVR12. There were no cases with reappearance of HBsAg during DAAs therapy. Among seven cases in the HBsAg group whose HBV DNA level was determined, HBV virological reactivation was detected in two subjects $(28.6 \%)$. Among all 11 subjects in the HBsAg group, there was one (9.1\%) case with HBV clinical reactivation, which was resolved following treatment with Entaclavir. The case with HBV clinical reactivation had a higher baseline HBV DNA viral load $(1,380 \mathrm{IU} / \mathrm{mL})$ compared with that of the other patients $(20-296 \mathrm{IU} / \mathrm{mL})$.

Manuscript submitted January 23, 2018, accepted February 27, 2018

aDivision of Gastroenterology, Department of Internal Medicine, Taichung Veterans General Hospital, Taichung, Taiwan, Republic of China

${ }^{b}$ Department of Internal Medicine, Chung Shan Medical University, Taichung, Taiwan, Republic of China

'Department of Internal Medicine, Yang-Ming University School of Medicine, Taipei, Taiwan, Republic of China

${ }^{\mathrm{d} C}$ Corresponding Author: Shou-Wu Lee, Division of Gastroenterology, Department of Internal Medicine, Taichung Veterans General Hospital, Taichung, 1650 Taiwan Boulevard, Sec. 4, Taichung 40705, Taiwan, Republic of China. Email: ericest429@yahoo.com.tw

doi: https://doi.org/10.14740/gr971w
Conclusion: HBV virological and clinical reactivation occurred in $28.5 \%$ and $9.1 \%$ of subjects with HBsAg seropositivity. No HBV reactivation was observed in the cases with past HBV infection.

Keywords: Hepatitis B; Hepatitis C; Direct-acting antivirals

\section{Introduction}

Hepatitic $\mathrm{C}$ virus (HCV) infection is a major worldwide disease. The current standard of care for subjects with $\mathrm{HCV}$ infection at all stages of the disease is interferon (IFN)-free combinations of direct-acting antivirals (DAAs) owing to their higher efficacy and low rate of adverse events. In areas with high-endemic hepatitis B virus (HBV), such as the Asia-Pacific region, it is vitally important to clarify the factors associated with $\mathrm{HBV}$ and $\mathrm{HCV}$ coinfection. According to past studies, the estimated prevalence of $\mathrm{HBV} / \mathrm{HCV}$ coinfection ranges from $5 \%$ to $20 \%$ in HBsAg-positive patients and from $2 \%$ to $10 \%$ in HCV-positive cases [1,2].

In HCV-infected patients who have undergone IFN and ribavirin (RBV) treatment, clearance of HCV was reported to induce reactivation of $\mathrm{HBV}$ and alanine aminotransferase (ALT) flares [3, 4]. Similarly, pan-oral DAAs treatment has been shown to be associated with HBV reactivation [5, 6]. The occurrence of these events recently prompted both the US Food and Drug Administration and the American Association for the Study of Liver Diseases (AASLD)/European Association for the Study of the Liver (EASL) to confirm the risk of HBV reactivation after DAA therapy [7].

However, the incidence and risk factors in individuals who received DAAs treatment and subsequently suffered from HBV reactivation remain unclear. The aim of this study was to investigate the rate of $\mathrm{HBV}$ reactivation in $\mathrm{HCV}$-infected patients who received pan-oral DAAs.

\section{Patients and Methods}

Data from subjects with chronic hepatitis $\mathrm{C}$ (CHC) who received pan-oral DAA therapy who visited the Medical Screening Center at Taichung Veterans General Hospital were ret- 
Table 1. The General Characteristics of Enrolled Cases

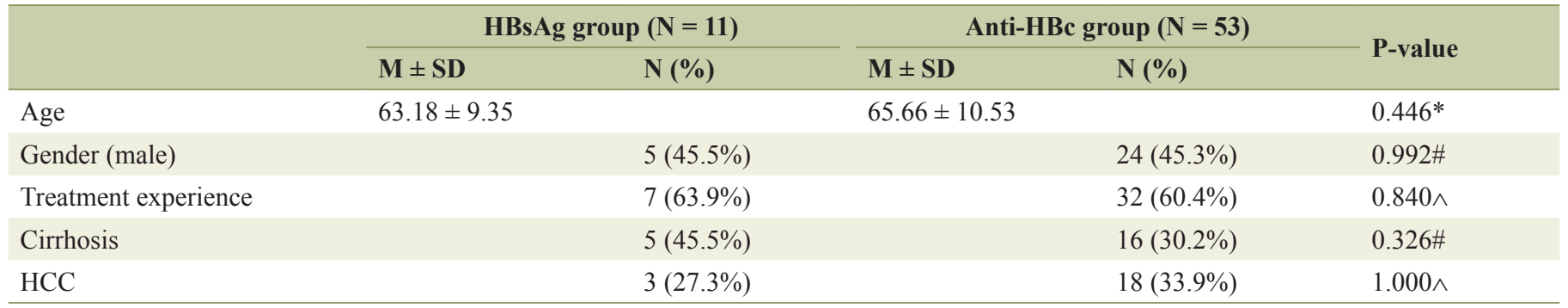

P-values were analyzed with Pearson's Chi-square test\#, Fisher's exact test $\wedge$, and $t$-test*. HCC: hepatocellular carcinoma; M: mean; N: number of patients; SD: standard derivation.

rospectively collected from October 2015 to May 2017. The general data of enrolled patients, including age, gender, $\mathrm{HCV}$ genotype (Roche, Cobas Amplicor Analyser, USA), HCV RNA (Roohe, Cobas TaqMan48, USA), cirrhosis status, hepatocellular carcinoma (HCC) status, and previous IFN treatment history, were recorded. Serum hepatitis B markers, including $\mathrm{HBsAg}$, anti-HBc, and $\mathrm{HBeAg}$, were collected.

The enrollment criteria were: 1) seropositivity for anti$\mathrm{HCV}$ longer than 6 months; 2) seropositivity for HBsAg or anti-HBc; 3) a completed pan-DAA therapeutic period. The exclusion criteria were: 1) previous DAAs treatment; 2) coinfection with human immunodeficiency virus (HIV) or other identified liver disease, such as autoimmune disease, Wilson's disease, or primary biliary cirrhosis.

The enrolled subjects were assigned to the HBsAg group, i.e., seropositive for $\mathrm{HBsAg}$, or the anti-HBc group, i.e., seropositive for serum anti-HBc Ab but seronegative for HBsAg.

Based on the current treatment guidelines and Taiwan's national insurance, DAA regimens such as sofosbuvir $(\mathrm{SOF})+$ ledipasvir (LDV), paritaprevir/ritonavir/ombitasvir + dasabuvir (Pro-D), and daclatasvir (DCV) + asunaprevir (ASV) were allowed to treat the subjects with $\mathrm{HCV}$ infection. The DAA regimens depended on the HCV genotype of each individual. In our study, the regimens for HCV genotype 1a-infected patients included SOF + LDV and Pro-D with RBV. For HCV genotype 1b-infected cases, the regmiens were SOF + LDV, Pro-D, and DCV + ASV. For HCV genotype 2 or other infections, the regimens included $\mathrm{SOF}+\mathrm{RAB}, \mathrm{SOF}+\mathrm{DCV}$, or SOF + LDV.

The serologic data, including HBV DNA, HCV RNA, ALT, and bilirubin, were recorded before and during the therapeutic period. Virologic response (VR) 4 was defined as 4 weeks after starting DAAs. End-of-treatment virologic response (ETVR) was defined as undetectable HCV RNA at the end of treatment. Sustained virologic response (SVR) 12 was defined as undetectable HCV RNA 12 weeks after completion of therapy [8]. HBV virologic reactivation was defined as a reappearance of $\mathrm{HBsAg}$, or increased $\mathrm{HBV}$ DNA by at least one $\log _{10} \mathrm{IU} / \mathrm{mL}$. HBV clinical reactivation was defined as virologic reactivation and serum ALT over two-fold of the upper limit of normal (male $>100 \mathrm{U} / \mathrm{L}$, female $>70 \mathrm{U} / \mathrm{L}$ ).

Data are expressed as standard deviation of the mean for each of the measured parameters. Gender, treatment experience, cirrhosis, HCC, and positive ratio of each stratified group are expressed as a percentage of the total patient number. Comparisons were made using Pearson's Chi-square test or Fisher's exact test to evaluate the contributions of gender and positive ratio of each stratified group. Independent $t$-test was used to analyze age and HCV viral load. A P-value $<0.05$ was considered statistically significant.

\section{Results}

Among all 153 subjects, there were $11(7.2 \%)$ cases with positive HBsAg and $53(34.6 \%)$ cases with anti-HBc but lack of HBsAg. They were labeled as the HBsAg group and the anti$\mathrm{HBc}$ group, respectively. The patients' general characteristics are shown in Table 1. The mean age of the two groups was similar (63.18 vs. 65.66 years), and female predominance $(54.5 \%$ vs. $54.7 \%$ ) was noted. Most individuals (60.4\% vs. $63.3 \%$ ) had past experience of IFN treatment. A portion of cases had cirrhosis $(45.5 \%$ vs. $30.2 \%)$ or HCC $(27.3 \%$ vs. $33.9 \%)$ in the HBsAg group and the anti-HBc group. All subjects that were seropositive for $\mathrm{HBsAg}$ were seronegative for $\mathrm{HBeAg}$ and seropositive for anti-HBe $\mathrm{Ab}$.

HCV status and the therapeutic efficacy of DAAs are displayed in Table 2. Most subjects had HCV genotype 1b-infection $(90.9 \%$ vs. $90.6 \%)$. A high HCV viral load with elevated serum ALT was found before DAAs treatment was started (mean HCV RNA 1.85 - $2.21 \times 10^{6} \mathrm{IU} / \mathrm{mL}$, mean ALT 67.55 $108.45 \mathrm{U} / \mathrm{L})$. The responses to DAAs in these two groups were optimal, with all individuals achieving ETVR and SVR12.

The serum ALT level of the anti-HBc group is shown in Figure 1. Most cases achieved normal ALT level after 1 month of DAAs treatment, and there was no biochemical breakthrough during the therapeutic period. Furthermore, among all 53 cases in the anti-HBc group, there was no case with reappearance of HBsAg during DAAs therapy.

Among all 11 cases in the HBsAg group, seven individuals had their HBV DNA level checked before and during DAAs treatment, and the HBV virus load is displayed in Figure 2. There were two subjects $(28.6 \%)$ with HBV virological reactivation during the DAAs treatment. Both of these patients had HCV genotype 1b-infection and received treatment with Pro-D. One case had HBV viral load from baseline $20 \mathrm{IU} / \mathrm{mL}$ to $3,000 \mathrm{IU} / \mathrm{mL}$ at the third month after treatment started, and another case had baseline $1,380 \mathrm{IU} / \mathrm{mL}$ to $14,000,000 \mathrm{IU} / \mathrm{mL}$ 
Table 2. HCV Status and the Therapeutic Efficacy of Direct-Acting Antivirals (DAAs) Treatment

\begin{tabular}{|c|c|c|c|c|c|}
\hline & \multicolumn{2}{|c|}{ HBsAg group $(\mathrm{N}=11)$} & \multicolumn{2}{|c|}{ Anti-HBc group $(N=53)$} & \multirow{2}{*}{ P-value } \\
\hline & $\mathrm{M} \pm \mathrm{SD}$ & N (\%) & $\mathbf{M} \pm \mathrm{SD}$ & $\mathbf{N}(\%)$ & \\
\hline HCV genotype & & & & & $0.310 \wedge$ \\
\hline $1 b$ & & $10(90.9 \%)$ & & $48(90.6 \%)$ & \\
\hline 2 & & $1(9.1 \%)$ & & $1(1.9 \%)$ & \\
\hline \multicolumn{6}{|l|}{ DAAs efficacy } \\
\hline VR4 & & $11(100 \%)$ & & $51(96.2 \%)$ & $1.000 \wedge$ \\
\hline ETVR & & $11(100 \%)$ & & $53(100 \%)$ & $1.000 \wedge$ \\
\hline SVR12 & & $11(100 \%)$ & & $53(100 \%)$ & $1.000 \wedge$ \\
\hline
\end{tabular}

P-values were analyzed with Fisher's exact test $\wedge$ and $t$-test*. ALT: alanine aminotransferase; DAAs: direct-acting antivirals; ETVR: End-of-treatment virologic response; M: mean; N: number of patients; SD: standard derivation; SVR: sustained virologic response; VR: virologic response.

at the second month after starting treatment.

The serum ALT of the HBsAg group is shown in Figure 3. Among all 11 subjects, there was only one (9.1\%) case with HBV clinical reactivation. This was a 53-year-old woman with a history of treatment-naive chronic HBV and HCV coinfection. Abdominal ultrasound showed no evidence of cirrhosis. Laboratory workup before DAAs treatment demonstrated ALT of $58 \mathrm{U} / \mathrm{L}$, HBV DNA of 1,380 IU/mL, HCV RNA of 670,000 $\mathrm{IU} / \mathrm{mL}$, and HCV genotyping Ib. Further evaluation disclosed that the patient was HbeAg-negative and anti-HBe Ab-positive. A combination of 3-month therapy with Pro-D was scheduled. Laboratory data at the first month revealed ALT of $15 \mathrm{U} / \mathrm{L}$, and undetectable HCV RNA. Similarly, laboratory workup at the second month disclosed undetectable HCV RNA, but ALT of $102 \mathrm{U} / \mathrm{L}$ and HBV DNA of 14,000,000 IU/mL. Treatment with Entaclavir $0.5 \mathrm{mg}$ per day for reactivated HBV was added. Laboratory data at the third month found undetectable HCV RNA, ALT of $68 \mathrm{U} / \mathrm{L}$, and HBV DNA of 2,130 IU/mL. Thereafter, positive SVR12 to HCV was achieved, with simultaneous ALT of $20 \mathrm{U} / \mathrm{L}$ and HBV DNA of $155 \mathrm{IU} / \mathrm{mL}$.

\section{Discussion}

Due to the shared modes of transmission, coinfection with both HBV and HCV is not uncommon. In China, where HBV

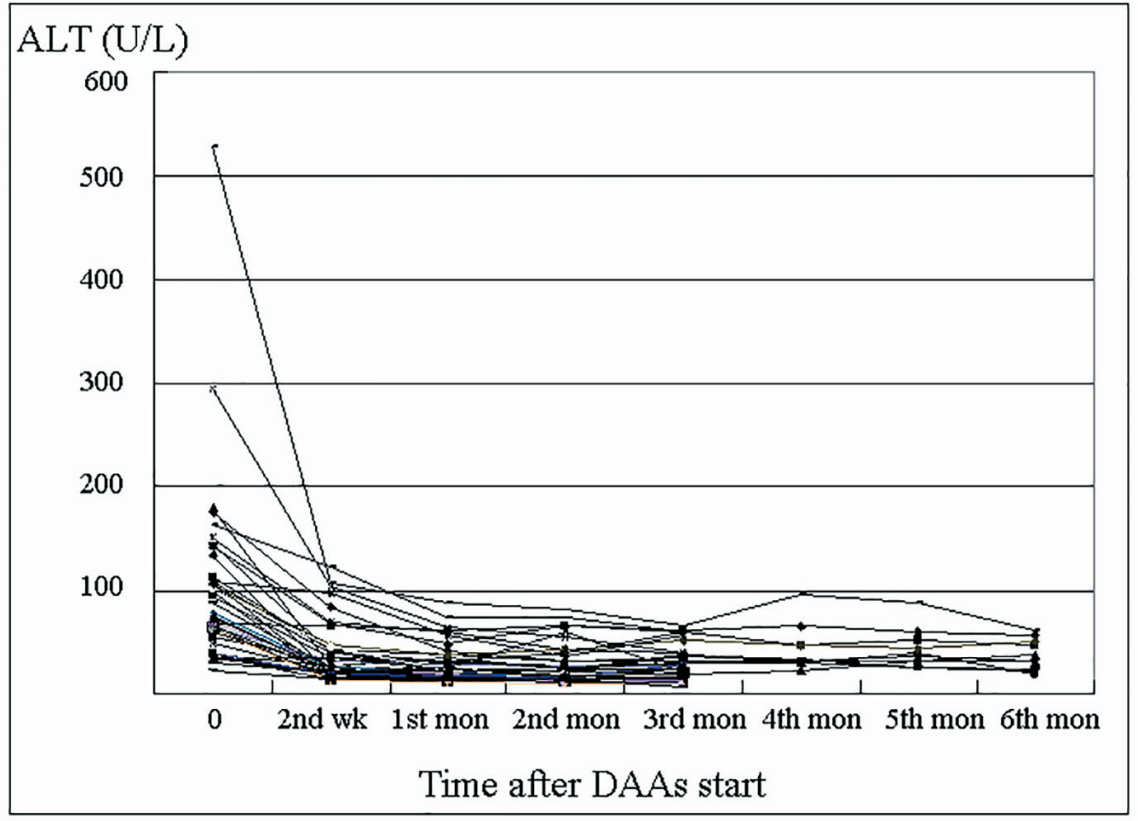

Figure 1. Changes in alanine aminotransferase (ALT) level of the anti-HBc group during direct-acting antivirals (DAAs) treatment. 


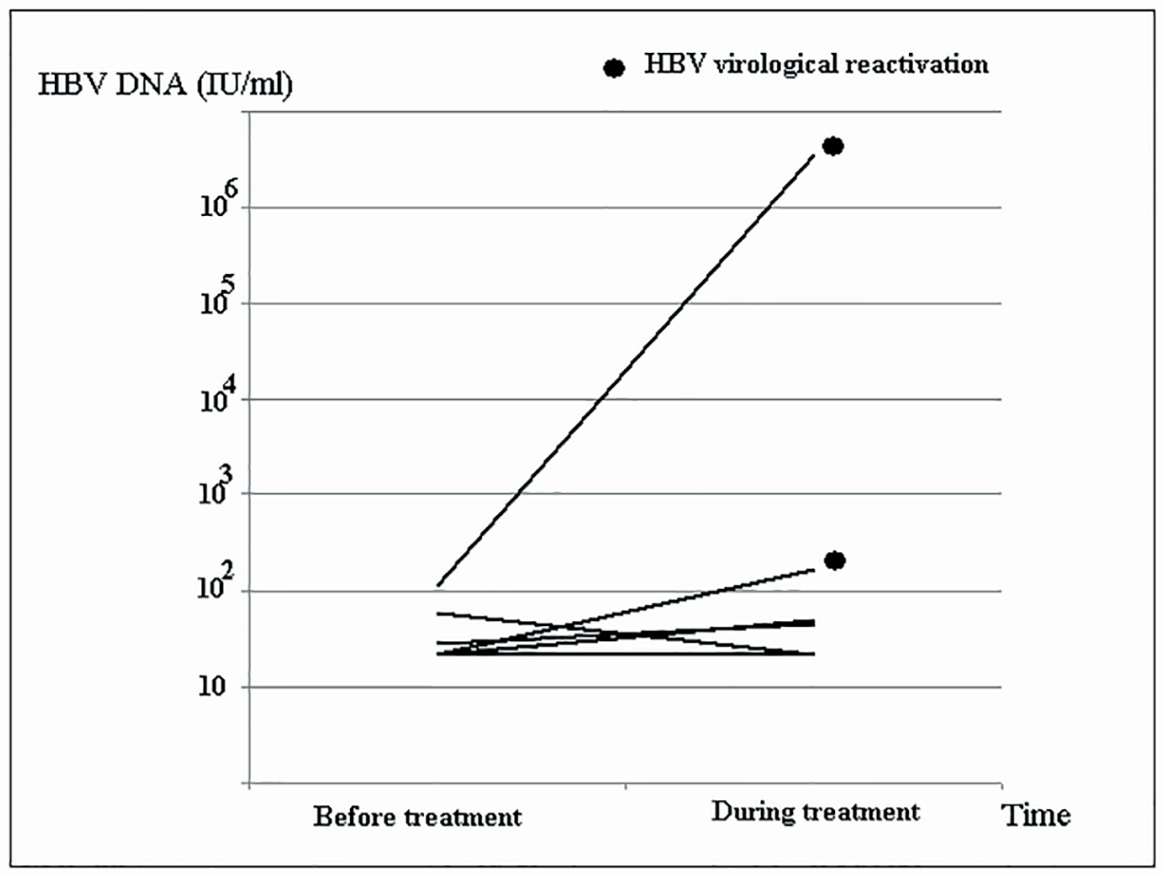

Figure 2. Changes in HBV DNA level of the HBsAg group during direct-acting antivirals (DAAs) treatment.

infection is endemic, $\mathrm{HBV}$ and $\mathrm{HCV}$ coinfection has been estimated to be as high as 8.4\% [9]. In the United States, between $30 \%$ and $60 \%$ of patients with $\mathrm{CHC}$ have serological evidence of previous exposure to $\mathrm{HBV}$, defined by the presence of anti$\mathrm{HBc}$ in the absence of $\mathrm{HBsAg}[2,10]$. Our results found $7.2 \%$ and $34.6 \%$ of patients with $\mathrm{HCV}$ infection were seropositive for $\mathrm{HBsAg}$ and anti-HBc, respectively.

According to previous studies, HBsAg carriers with concurrent HCV infection have lower HBV viremia, lower levels of HBsAg in serum, and lower levels of intrahepatic HBsAg

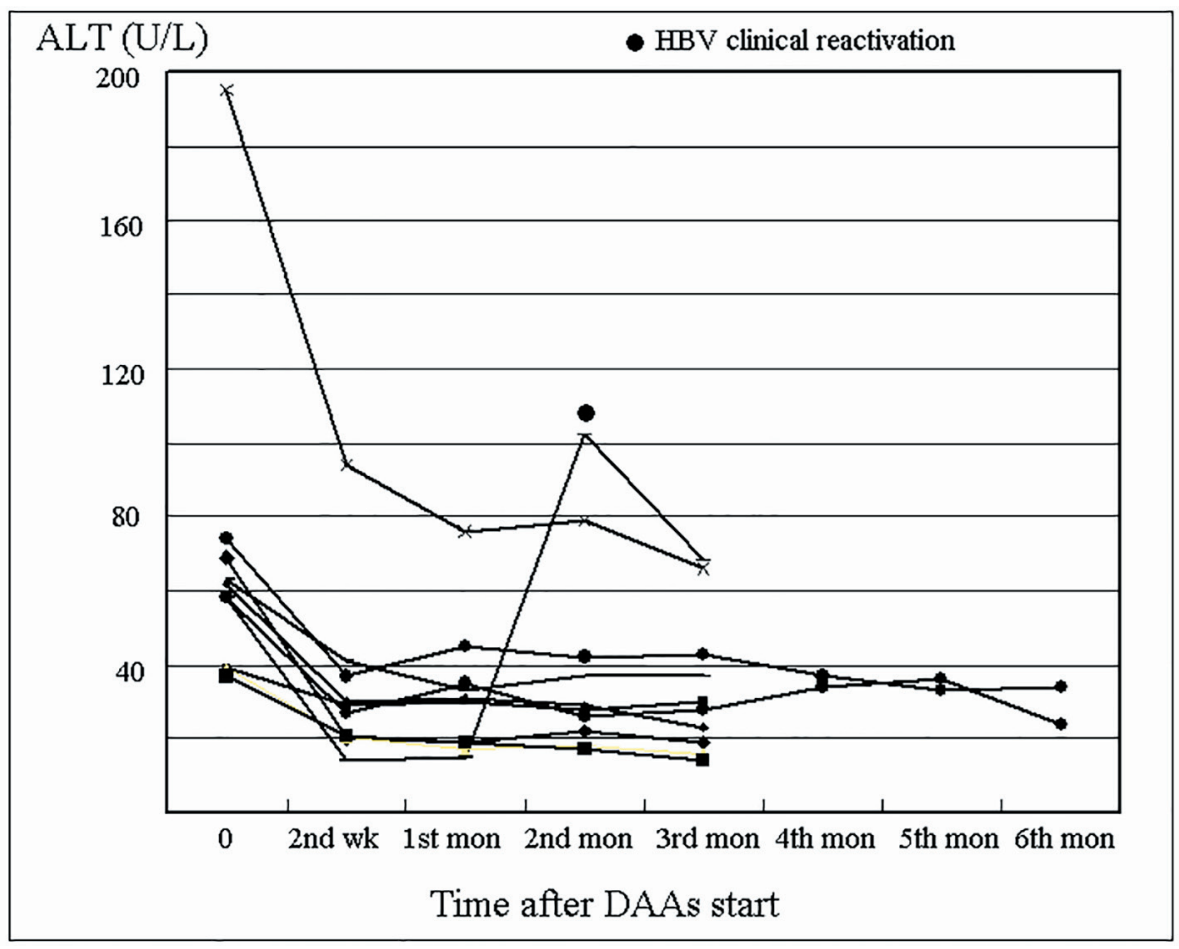

Figure 3. Changes in alanine aminotransferase (ALT) level of the HBsAg group during direct-acting antivirals (DAAs) treatment. 
$[11,12]$. These findings suggest that HCV infection can suppress HBV replication. Moreover, HCV superinfection in the setting of chronic hepatitis B can result in HBeAg seroconversion [9]. Our study revealed a similar finding. In our cases that were seropositive for $\mathrm{HBsAg}$, the mean HCV RNA of all 11 subjects was $1,850,000 \mathrm{IU} / \mathrm{mL}$, and the mean HBV DNA of seven subjects was $256.71 \mathrm{IU} / \mathrm{mL}$. All our enrolled cases were seronegative for $\mathrm{HBeAg}$.

Suppression or clearance of HCV infection with effective anti-HCV therapy using either IFN- or DAA-based therapy ceases the suppression of $\mathrm{HBV}$ replication, thereby resulting in $\mathrm{HBV}$ reactivation. Clearance of $\mathrm{HCV}$ by IFN leading to induction of HBV reactivation has been previously reported $[3,4]$. Because IFN also exerts an inhibitory effect on HBV replication [13], the additional inhibitory effect of IFN on HBV probably ameliorates HBV reactivation and delays its occurrence, which mostly occurs after stopping therapy.

To date, IFN-free DAA combination therapy has been recommended as the first line therapy for CHC. Thus, there is increasing concern regarding hepatitis flare related to $\mathrm{HBV}$ reactivation during DAA therapy in $\mathrm{CHC}$ patients with current or past $\mathrm{HBV}$ infections. According to a number of previous case reports and series, the severity of hepatitis after pan-oral DAAs treatment ranged from HBV reactivation without hepatitis to fulminant hepatic failure requiring liver transplantation $[5,6,14]$.

In a recent meta-analysis, the incidence rates of $\mathrm{HBV}$ reactivation and associated hepatitis after DAAs treatment were $14.5 \%$ and $12.5 \%$, respectively. The rate of $\mathrm{HBV}$ reactivation was similar between DAAs and IFN-based therapy (14.5\% vs. $12.2 \%$ ), but the rate of hepatitis was higher with DAA-based therapy $(12.5 \%$ vs. $0 \%)$. Furthermore, HBV reactivation was reported to occur mostly at 4 - 12 weeks during DAA treatment, which was earlier than with IFN-based therapy [7].

A cohort study in Hong Kong found three (30\%) of the 10 HbsAg-positive patients experienced a hepatitis flare, and none of the 124 patients with occult HBV infection had a hepatitis flare related to $\mathrm{HBV}$ reactivation [15]. Another cohort study in Taiwan found four $(57.1 \%)$ and one $(14.2 \%)$ of the seven subjects had HBV virological and clinical reactivation, respectively. No episodes of HBV virological reactivation were observed among the cases with a past HBV infection [16].

Our results showed no HBsAg seroreversion occurred in the 53 cases with positive anti-HBc after DAA therapy. In the 11 individuals with HBsAg seropositivity, two (28.5\%) of the seven patients and one $(9.1 \%)$ of the 11 patients had virologic and clinical reactivation, respectively. $\mathrm{HBV}$ reactivation in the two cases occurred in the second and third months during DAAs treatment. HBV clinical reactivation was resolved following treatment with oral Enteclavir. The lower rates of HBV reactivation in our study might be due to different definitions of HBV virologic and clinical reactivation in our report compared with those applied in other studies. Furthermore, in our study, HBV virological load was not checked in every case or was not periodically monitored, which might have resulted in an underestimation of the rates of HBV reactivation.

Similarly, our patients with past HBV infection, as in other studies $[7,15,16]$, who presented with anti-HBc, did not have a risk of $\mathrm{HBV}$ reactivation during the DAAs therapeutic period. However, there have been cases reported in the literature whose HBV infection had been resolved and yet subsequently experienced HBV reactivation after DAAs treatment, with one patient requiring liver transplantation $[5,6,17]$. Although rare, $\mathrm{HBV}$ reactivation due to DAAs treatment in patients with previously resolved HBV infection may occur and therefore physicians should be aware of this possibility in certain patient populations.

The risk factors of HBV reactivation during DAAs treatment have yet to be fully elucidated. Different DAAs regimens are available, including SOF-based therapy, DCV/ASV, and Pro-D [5, 16-18]. In our study, the regimen for cases with HBV reactivation was Pro-D in most of our enrolled cases because of genotype $1 \mathrm{~b}$-infection and the limitation of national health insurance coverage in Taiwan. The association of different DAA regimens and HBV reactivation warrants further investigation.

In our cases with HBV clinical reactivation, a higher baseline HBV DNA viral load $(1,380 \mathrm{IU} / \mathrm{mL})$ compared with that of the other cases $(20$ - $296 \mathrm{IU} / \mathrm{mL})$ was noted. However, previous studies reported cases with HBV reactivation had baseline HBV DNA ranging from undetectable to a high level [5, 16-18]. Owing to the heterogenous nature of the results, it was not possible to make a definitive conclusion about the role of baseline HBV viral load in HBV reactivation [7].

There were several limitations in our study. First, this study used a retrospective design and was conducted in a single tertiary care center. Selection bias might have existed and therefore our sample of patients may not be representative of the entire population. Second, the small number of patients in our study might have led to unreliable estimations in the results. Third, the lack data on regular HBV viral load might have led to an underestimation of the rate of HBV virological reactivation. Finally, the quantitative $\mathrm{HBsAg}$ data during DAA treatment were not measured in our study. Further research with analysis of more variables is needed.

In conclusion, our study found $\mathrm{HBV}$ virological and clinical reactivation occurred in $28.5 \%$ and $9.1 \%$ of the subjects with seropositivity for HBsAg. No HBV reactivation was observed in the cases with past HBV infection.

\section{References}

1. Caccamo G, Saffioti F, Raimondo G. Hepatitis B virus and hepatitis $\mathrm{C}$ virus dual infection. World J Gastroenterol. 2014;20(40):14559-14567.

2. Bini EJ, Perumalswami PV. Hepatitis B virus infection among American patients with chronic hepatitis $\mathrm{C}$ virus infection: prevalence, racial/ethnic differences, and viral interactions. Hepatology. 2010;51(3):759-766.

3. Chu CJ, Lee SD. Hepatitis B virus/hepatitis C virus coinfection: epidemiology, clinical features, viral interactions and treatment. J Gastroenterol Hepatol. 2008;23(4):512520.

4. Gordon SC, Sherman KE. Treatment of HBV/HCV coinfection: releasing the enemy within. Gastroenterology. 2009;136(2):393-396.

5. Collins JM, Raphael KL, Terry C, Cartwright EJ, Pillai A, Anania FA, Farley MM. Hepatitis B virus reactivation 
during successful treatment of hepatitis $\mathrm{C}$ virus with sofosbuvir and simeprevir. Clin Infect Dis. 2015;61(8):13041306.

6. Ende AR, Kim NH, Yeh MM, Harper J, Landis CS. Fulminant hepatitis B reactivation leading to liver transplantation in a patient with chronic hepatitis $\mathrm{C}$ treated with simeprevir and sofosbuvir: a case report. J Med Case Rep. 2015;9:164.

7. Chen G, Wang C, Chen J, Ji D, Wang Y, Wu V, Karlberg $\mathrm{J}$, et al. Hepatitis B reactivation in hepatitis B and C coinfected patients treated with antiviral agents: A systematic review and meta-analysis. Hepatology. 2017;66(1):1326.

8. Martinot-Peignoux M, Maylin S, Moucari R, Ripault MP, Boyer N, Cardoso AC, Giuily N, et al. Virological response at 4 weeks to predict outcome of hepatitis $\mathrm{C}$ treatment with pegylated interferon and ribavirin. Antivir Ther. 2009;14(4):501-511.

9. Yu G, Chi X, Wu R, Wang X, Gao X, Kong F, Feng X, et al. Replication inhibition of hepatitis $\mathrm{B}$ virus and hepatitis $\mathrm{C}$ virus in co-infected patients in Chinese population. PLoS One. 2015;10(9):e0139015.

10. Tyson GL, Kramer JR, Duan Z, Davila JA, Richardson PA, El-Serag HB. Prevalence and predictors of hepatitis $B$ virus coinfection in a United States cohort of hepatitis C virus-infected patients. Hepatology. 2013;58(2):538545.

11. Crockett SD, Keeffe EB. Natural history and treatment of hepatitis $\mathrm{B}$ virus and hepatitis $\mathrm{C}$ virus coinfection. Ann Clin Microbiol Antimicrob. 2005;4:13.

12. Sato S, Fujiyama S, Tanaka M, Yamasaki K, Kuramoto I, Kawano S, Sato T, et al. Coinfection of hepatitis C virus in patients with chronic hepatitis B infection. J Hepatol. 1994;21(2):159-166.

13. Lau GK, Piratvisuth T, Luo KX, Marcellin P, Thongsawat S, Cooksley G, Gane E, et al. Peginterferon Alfa-2a, lamivudine, and the combination for $\mathrm{HBeAg}$-positive chronic hepatitis B. N Engl J Med. 2005;352(26):2682-2695.

14. Hayashi K, Ishigami M, Ishizu Y, Kuzuya T, Honda T, Nishimura D, Goto H, et al. A case of acute hepatitis B in a chronic hepatitis $\mathrm{C}$ patient after daclatasvir and asunaprevir combination therapy: hepatitis $\mathrm{B}$ virus reactivation or acute self-limited hepatitis? Clin J Gastroenterol. 2016;9(4):252-256.

15. Wang C, Ji D, Chen J, Shao Q, Li B, Liu J, Wu V, et al. Hepatitis due to reactivation of hepatitis $B$ virus in endemic areas among patients with hepatitis $\mathrm{C}$ treated with direct-acting antiviral agents. Clin Gastroenterol Hepatol. 2017;15(1):132-136.

16. Yeh ML, Huang CF, Hsieh MH, Ko YM, Chen KY, Liu TW, Lin YH, et al. Reactivation of hepatitis B in patients of chronic hepatitis $\mathrm{C}$ with hepatitis $\mathrm{B}$ virus infection treated with direct acting antivirals. J Gastroenterol Hepatol. 2017;32(10):1754-1762.

17. Takayama H, Sato T, Ikeda F, Fujiki S. Reactivation of hepatitis B virus during interferon-free therapy with daclatasvir and asunaprevir in patient with hepatitis B virus/hepatitis C virus co-infection. Hepatol Res. 2016;46(5):489-491.

18. Hwang JP, Somerfield MR, Alston-Johnson DE, Cryer DR, Feld JJ, Kramer BS, Sabichi AL, et al. Hepatitis b virus screening for patients with cancer before therapy: American society of clinical oncology provisional clinical opinion update. J Clin Oncol. 2015;33(19):2212-2220. 